\title{
INTENSIDAD DE USO DE LA TIERRA Y TASAS DE OCUPACION GANADERA EN LA PRADERA PAMPEANA (1816-1852).
}

\author{
JUAN CARLOS GARAVAGLIA(*)
}

\section{Introducción}

En su perspectiva histórica, el problema de la intensidad del uso ganadero de la tierra en la pradera pampeana es absolutamente central en todo análisis de la economía agraria del siglo XIX. Hasta ahora, y quien escribe estas líneas no ha sido una excepción, los historiadores hemos acudido a tasas de ocupación o de receptividad ganadera muy generales para utilizarlas en todo período y en todas las subregiones de la pradera pampeana ${ }^{1}$. Este sistema no nos satisface ya, pues el avance historiográfico de los últimos años exige hoy un análisis mucho más afinado. Es justamente en función de ese análisis mucho más fino que hemos escrito las páginas que siguen. Esperamos que el lector, sabiendo cuáles son los objetivos que nos hemos planteado, podrá disculpar la aridez que campea en ellas...

\section{Metodología y fuentes}

Hemos utilizado los inventarios post mortem para calcular las tasa mínimas reales de ocupación (es decir, las tasas mínimas efectivamente en uso en el momento del inventario). De un total de 318 inventarios de bienes rurales de las estancias ${ }^{2}-0$ sea, no hemos to-

- Ecole des Hautes Etudes, Paris. (e-mail: gara@ehess.fr)

1 Ver: Azcuy Ameghino y Martínez Dougnac, 1989; Amaral y Ghío, 1990; Garavaglia, 1993; Canedo, 1997.

2 Hasta bastante más allá de fines del segundo período, es decir, hasta los años sesenta 
mado en cuenta a las chacras- que poseemos para el período 1816/1852, hemos utilizado exclusivamente, por razones obvias, los inventarios de aquellos productores que eran propietarios o enfiteutas de tierras (si no fuese así, no tendríamos la superficie del establecimiento y no podríamos calcular las tasas mínimas efectivas de ocupación o de receptividad). Señalemos que los propietarios son el 53 \% del total de esos 318 inventarios, es decir, 162 inventarios. Pero, de esta masa de 162 inventarios, debemos restar todos aquellos cuyos datos acerca de la superficie no son completamente fiables ${ }^{3}$ y ello nos deja una cifra menor. La cantidad total de establecimientos concernidos -en este artículo, la unidad no es el inventario sino el establecimiento productivo por razones más que obvias- es de 153 estancias.

Las estancias que hemos podido mensurar con certeza están entonces distribuidas en forma desigual de acuerdo a las regiones. Recordemos que en lo que se refiere a la cifra total de 318 inventarios la distribución era la siguiente: tenemos el Norte ${ }^{5}$ con 34 inventarios; Areco $0^{6}$ posee 56, Luján tiene 62 inventarios y las estancias de cercanías ${ }^{8}$ con 32 . Vie-

del XIX, la palabra "estancia" seguía manteniendo un sentido claro de "explotación pecuaria", sin que hubiese adquirido aún el tinte nobiliario que tendría después. Si alguna duda le queda al lector acerca de ello, la lectura de las discusiones surgidas alrededor de la redacción del Código Rural (Antecedentes, 1864) son en ese sentido claramente representativas. Aquí "estancia" es en general una explotación pecuaria, en forma completamente independiente de su tamaño (uno de los participantes en la discusión no duda en llamar pequeña estancia ¡a una explotación de 30 ha.!), de sus condiciones de propiedad y de su variada vocación productiva. Es estrictamente en esta acepción que utilizaremos la palabra en el texto.

3 Cuando, por ejemplo, las medidas son dadas en varas frontales y no hay total seguridad acerca de la medida de los fondos. O directamente, cuando no se especifican medidas porque la tierra no ha sido mensurada todavía, o lo ha sido defectuosamente, etc.

\section{Ver Garavaglia, 1997.}

5 Este incluye según el censo de 1838, en Archivo General de la Nación (en adelante: AGN), sala IX-25-6-2, a los partidos de San Nicolás de los Arroyos, Arrecifes, Baradero, Pergamino, Rojas, Salto y San Pedro. La cantidad de inventarios es aquí bastante pobre y el nivel de representatividad de estos datos es bajo.

6 Los partidos de San Antonio de Areco, Fortín de Areco, San Andrés de Giles y Exaltación de la Cruz según su extensión en 1838.

7 Este comprende los partidos de Luján, Pilar, Guardia de Luján (con Chivilcoy) de acuerdo a la división de 1838.

8 Corresponde a los partidos de Quilmes, Matanza, San José de Flores, Las Conchas, San Fernando y San Isidro. 
ne después Sur l, que comprende a todos los partidos que se hallaban al sur y suroeste en las tierras al interior del Río Salado ${ }^{9}$ y Sur // para todos aquéllos ${ }^{10}$ que se extienden al exterior del Salado, frontera natural entre la pampa ondulada y la pampa deprimida. En el primero de los dos partidos sureños tenemos 102 inventarios y en el segundo contamos con 32 inventarios. Por supuesto, estos inventarios incluyen algunas veces más de un establecimiento.

Ahora bien, en lo que hace a nuestras 153 estancias, la distribución es la siguiente: el Norte cuenta con 19 estancias; Areco posee 22 establecimientos y Luján 42; las estancias de cercanías tienen 9 establecimientos. Sur I alberga 49 y Sur II alcanza a 12 estancias. Como se puede observar, los datos de Luján, Norte y Sur I son los más representativos, siendo la representatividad de Areco y Sur /I menor, pero fiable; en el caso de las estancias de cercanías es bastante baja. Lógicamente, la representatividad está también en relación al porcentaje de propietarios sobre el total regional. Señalemos cuál era el porcentaje de no propietarios por cada una de las regiones: Norte $41 \%$, Areco $52 \%$, Luján $29 \%$, estancias de cercanías $50 \%$, Sur $149 \%$ y finalmente Sur $1 / 47 \%$.

En este estudio convertimos todas las medidas a hectáreas y hemos acudido a las siguientes equivalencias. Partiendo de las varas lineales, el cálculo de la superficie es relativamente simple: se multiplican las varas del frente y el fondo $(200 \times 9.000=18.000$ varas cuadradas), este resultado se divide por $1,3334^{11}(18.000 \% 1,3334=13.499,325)$ y éste a su vez, se divide por 10.000 para obtener finalmente las hectáreas $(13.499,325 \% 100=$ $134,99325 \mathrm{ha}$ ). Recordemos que la legua lineal posee 6.000 varas. En los casos que la medida está determinada en leguas cuadradas, hemos tomado la cifra de 2.700 hectáreas para

9 Es decir, Lobos, Navarro, Cañuelas, San Vicente, Ensenada, Magdalena, Chascomús, Ranchos y Monte según los límites del año 1838. Pero, hay que señalar que en todos los partidos fronterizos del Río Salado, es evidente que hay propietarios que explotan campos "avanzados" hacia el otro lado del Río Salado.

10 En 1838, éstos eran: Dolores, Monsalvo, Azul, Tapalqué y Fuerte Independencia (Tandil), con el agregado de Bahía Blanca y Carmen de Patagones -hay que recordar que la frontera no incluye realmente a estas dos últimas poblaciones que se hallan casi completamente aisladas por tierra. En 1854, los partidos han crecido sensiblemente y ahora son: Pila, Dolores, Tordillo, Las Flores, Ajó, Vecino, Lobería, Mar Chiquita, Tandil, Saladillo, Tapalqué, Azul, Tuyú, Bahía Blanca y Carmen de Patagones.

11 Divisor que se utiliza para pasar de un sistema hexagesimal a otro decimal. 
la legua, confirmada por la propia documentación (6.000 varas x 6.000 varas da exactamente $2.699,865 \mathrm{ha}$ ). Las cuadras lineales poseen 150 varas (la vara mide 0,8666 metros) y en cuanto a las cuadras cuadradas, resultan generalmente de 150 por 150 varas, es decir, 1,6874 ha (de este modo, una legua lineal tiene 40 cuadras lineales y la legua cuadrada 1.600 cuadras cuadradas). En un solo caso, hemos hallado una suerte de estancia y justamente en esa ocasión -se trata de una "suerte" en el Azul- se nos dan las medidas exactas: esta "suerte" poseía 2.025 ha"; se trata aquí de una "suerte de estancia" resultado de un muy peculiar proceso ${ }^{13}$, pero, en general se acepta esta cifra para ella y siempre inferior a la legua cuadrada ${ }^{14}$.

Es obvio que todas estas medidas tienen exclusivamente -hasta demostración en contrario- una validez regional y están relacionadas con la calidad y el tipo de uso de la tie$\mathrm{rra}^{15}$. Los datos de Latzina, recogidos a fines de la década del ochenta -y que se hallaban aún en pleno uso pese a la ley del 13 de julio de 1877 que estableció el sistema métrico decimal en toda la república- muestran una clara separación en tres áreas: la región pampeana, con la legua de 6.000 varas de 0,866 metros, el área de influencia cuyana con la legua de 6.000 varas de 0,836 metros y finalmente, los casos particulares de Tucumán y Santiago del Estero con la legua de 5.000 varas de 0,866 y 0,8673 metros, respectivamente $^{16}$.

12 Sucesión de Bernardino Muga, 1840, AGN-Suc. 6799.

13 Sobre proceso de reparto de "suertes" en el Azul, ver Infesta, 1994.

14 En efecto, ésta gira entre 1.875 y 2.025 ha. Ver Giberti, 1981. La cifra de 1.875 ha. de Giberti se refiere a la legua cuadrada, pero calculada sobre 5.000 varas, como efectivamente medía la legua en algunas regiones (tal era el caso de Santiago del Estero). José María Jurado (1875: 34) da para la "suerte de estancia" una medida de $3 / 4$ de legua cuadrada, es decir un total de 2.025 ha.; esta misma medida es la aceptada en las discusiones casi contemporéneas acerca del Código Rural, y en Beaumont, 1957: 118.

15 Así es, como dijimos en la nota precedente, que una legua santiagueña posee 5.000 varas (pero, su "suerte de estancia" medía generalmente 4 leguas cuadradas, es decir casi 7.500 ha.), una cuadra cuadrada tucumana tiene 2,066 ha. y por el contrario, la "suerte de estancia" oriental es idéntica a la bonaerense; en efecto, ver Informe, 1958: 82-84.

16 Según los datos de Latzina, la legua poseía casi la misma medida en Buenos Aires, Santa Fe, Corrientes, Entre Ríos, Córdoba y San Luis -con una pequeña diferencia en estas tres últimas. Ver Latzina, 1888. 
Como se verá, hemos confeccionado una serie de mapas para poder comparar las tasas mínimas efectivas por regiones en dos períodos diversos, 1816/1829 y 1836/1852. Estos mapas han sido realizados a partir de la utilización de una escala diferencial por tipo de ganado: hemos otorgado una cifra de 10 puntos por hectárea a los vacunos y yeguarizos (es sabido que éstos ocupan un poco más de terreno que aquéllos, pero hemos preferido no complicar demasiado esa escala, ya bastante aventurada) y le hemos dado dos puntos a los ovinos. De este modo, hemos podido calcular porcentajes diferenciales de ocupación o de receptividad por hectárea para las regiones y los períodos a los efectos de poder representarlos en los mapas que hemos confeccionado.

\section{Las tasas de ocupación}

Hablamos aquí de "tasa de ocupación" y obviamente ella es un sinónimo de "receptividad ganadera" o "carga ganadera". Además, una advertencia: decimos tasas mínimas efectivas ¿y por qué "mínimas"? Porque indudablemente, hay que tener en cuenta el fenómeno del arriendo", de los "pobladores" y de la "agregación"18; esos diversos tipos de formas de ocupación del suelo, que presuponen la propiedad de algunos animales y que hemos Ilamado "pastores puros" en otro estudio", falsean bastante los datos en las áreas en los cuales el porcentaje de propietarios es más bajo. Los productores que no son propietarios de la tierra ocupan parcialmente los campos que muchas veces tienen dueño y con cierta frecuencia corresponden a nuestros titulares de inventarios (y es evidente que los bienes de los arrendatarios no estarán inventariados junto con los de los causantes). Por lo tanto, las tasas de ocupación que nosotros hemos calculado en las áreas en donde el arriendo es importante, son las mínimas posibles.

17 Hay hoy algunos trabajos excelentes sobre el tema, ver, por ejemplo, Fradkin, 1995.

18 Algunos de los ganaderos que participan en las discusiones acerca de la redacción del Código Rural, lo dicen con todas las letras "El infrascripto cree deber llamar la atención de la Comisión sobre ese inmenso número de agregados que hay en la campaña, no sólo en los que poseen un pequeño campo, tanto más perjudiciales, sino en los establecimientos de mayor extension, resultando que el que més tiene no alcanza a cien cabezas..." , respuesta de Venancio Casalins de Ranchos (Antecedentes, 1864: 52-53). Otro afirma "Además, como hay propietarios que acostumbran llenar, materialmente, su campo de poblaciones, ya sea en arrendamiento ó por gracia...", Ignacio Correas de Buenos Aires (idem: 67).

19 Ver Garavaglia, 1997. 
La primer sorpresa surge cuando tomamos los datos generales por regiones; es decir, los datos regionales no diferenciados por períodos. Si las tasas de ocupación que habíamos utilizado en nuestros estudios y que no se alejaban mucho de las que usaban diversos autores (es decir, un vacuno o yeguarizo cada hectárea y media y un ovino cada media hectárea) se aproximan burdamente a las tasas mínimas efectivas para las regiones "nuevas", ellas son casi inútiles en los casos de las áreas de más vieja colonización y por supuesto, no tienen la menor flexibilidad en el análisis temporal. Veamos los datos generales (recordemos que la superficie está dada en hectáreas):

\begin{tabular}{|lllllll|}
\hline \multicolumn{6}{c|}{ Cuadro 1 / Ocupación ganadera por regiones: } & $\mathbf{1 8 1 6 / 1 8 5 2}$ \\
& Norte & Areco & Luján & Est.Cercs. & Sur I & Sur II \\
superficie & 37614 & 15862 & 64295 & 8544 & 250753 & 185418 \\
número ests. & 19 & 22 & 42 & 9 & 49 & 12 \\
vac/yeguariz. & 36842 & 17283 & 55828 & 5760 & 120172 & 88150 \\
ovinos & 19376 & 14633 & 64524 & 11267 & 71763 & 16226 \\
\hline
\end{tabular}

Como se puede comprobar, en la mayor parte de las regiones, las tasas de ocupación son mucho más altas que las que usualmente hemos utilizado y como decíamos más arriba, ellas sólo sirven para las áreas "nuevas" y aún en ese caso, si nos referimos a los diversos períodos, veremos que hay cambios de importancia. Primera constatación general: en los partidos de vieja colonización, las tasas usuales de ocupación -para los propietarios de las tierras o para los enfiteutas- son mucho más altas que las supuestas y llegamos a tasas "modernas", es decir de un gran animal por hectárea, más una cantidad variable de ovinos, oscilando de uno a seis. Si volvemos a señalar que en algunas de estas mismas áreas de vieja colonización los no propietarios son abundantes (tal el caso de Areco, de las estancias de cercanias y de las dos áreas Sur I y Sur $\left./ I^{20}\right)$, las tasas reales debieron haber sido más altas aún. Otro hecho que ya hemos señalado en varias ocasiones, pero conviene ahora volver a mostrar: los promedios por hectárea de los establecimientos de la muestra están bien lejos de las cifras fabulosas que algunos han supuesto. Si son 15.800 ha. en Sur II y 5.048 ha. en Sur l, bajan a 1.980 ha. en el Norte, a 1.531 ha. en Luján, a sólo 949 ha. en las estancias de cercanías y a un magro 721 ha. en el caso de Areco. Y aquí estamos hablando sólo de los propietarios...

20 Recordemos los porcentajes de no propietarios en esas cuatro áreas: Areco $52 \%$, estancias de cercanias $50 \%$, Sur / $49 \%$ y Sur $1 / 47 \%$.

10 
Pero, sólo comprenderemos la importancia de estas cifras al pasar al análisis por períodos, siempre conservando la distinción por regiones. ¿Cómo elegir la "buena" cronología? Y bien, pensamos que la mejor forma de ordenar los períodos esté relacionada con los efectos de la gran sequía de 1828/1832, una de las peores en la historia de la campaña hasta ese entonces.

Según el médico Francisco J. Muñiz -que en esos momentos vivía en el pueblo de Luján- más de dos millones de animales murieron en esos años ${ }^{21}$ y aún cuando el oeste y el norte parecen haber sido más tocados (una recorrida por los juzgados de paz al norte de Buenos Aires en estos años nos muestra el impacto de esta gran sequía 22 , muchas otras fuentes confirman ese dato para todas las regiones de la campaña ${ }^{23}$. Y por supuesto, como lo señala el propio Juan Manuel de Rosas en 1831, ricos y pobres no se enfrentan de igual modo a las consecuencias de la sequía ${ }^{24}$. Las cifras relativas a las tasas de ocupación no ha-

21 Ver sus "Apuntes topográficos del territorio y adyacencias del Departamento del Centro de la provincia de Buenos Aires, con algunas referencias a lo demés de su campaña", en Muñiz, s/f.

22 En mayo de 1831, el Juez de Paz de Areco dice que la “...terrible calamidad de la seca padecida... ha hecho que los animales salten las zanjas de las quintas en busca de pasturas -los quinteros, ni lerdos ni perezosos, la emprenden a balazos o a punta de chuza con los intrusos... (Juez de Paz sustituto de San Antonio de Areco, 31/5/1831, AGN-X-21-5-7); el Juez de Baradero afirma que no han quedado más de "...dos estancias donde sacar escasamente... treinta cabezas de ganado para remitir al ejército (Juzgado de Baradero, 31/5/1831, AGN-X-20-10-3). En Exaltación de la Cruz, se ordena en octubre de ese mismo año no proceder a la yerra por la confusión de ganados "...originada en la actual seca..." (Juez de Paz de Exaltación de la Cruz, 4/10/1831, AGN-X-21-1-3). En febrero de 1832, el Juez de Pilar dice “...estan los campos de esta Sesion aridos y desiertos por la cruel epidemia que nos aflige..." y en agosto de 1832, el Juez de Paz de Arrecifes expone que "...con motivo de la horrosa epidemia havían quedado algunas Familias del todo insolventes..." (Juzgado de Paz de Pilar, 1/12/1832, AGN-X-21-4-1 y Juez de Paz de Arrecifes, carta del 26/1/1833, recordando una del 10/8/1832, en AGN-X-20-9-7).

23 En 1833, la mayor parte de los grandes productores del oeste y norte de la campaña aparecen casi sin rodeos, tal como lo señala una fuente de ese año, confirmando las aseveraciones de Muñiz (AGN-X-16-3-3). Y si bien la sequía parece haber sido más fuerte en el norte que en el sur, también esta región fue tocada. En 1832, el Juez de Paz de Dolores informa que hasta las espadañas se habían secado en su partido, AGN-X-21-1-2. Las cartas entre los encargados de las estancias de Anchorena ubicadas en Camarones y Averias (en plena región Sur II), nos muestran los efectos devastadores de la sequía sobre los ganados, AGN-VII-4-4-2 y 4-4-3.

24 En una carta escrita en mayo de 1831 desde la Hacienda de Figueroa -y que no tiene 
cen más que confirmar este hecho, permitiéndonos además una mejor regionalización del fenómeno del impacto de esa gran sequia. Comencemos entonces por el período previo a la sequía:

\begin{tabular}{|llllll|}
\hline \multicolumn{5}{|c|}{ Cuadro 2 / Ocupación ganadera por regiones: } & 1816/1829 \\
& Norte & Areco & Luján & Est.Cercs. & Sur I \\
superficie & 7954 & 9966 & 31852 & 2089 & 158907 \\
número ests. & 7 & 16 & 26 & 3 & 24 \\
vac/yeguariz. & 7577 & 14312 & 28934 & 2144 & 74709 \\
ovinos & 3097 & 12857 & 40497 & 4069 & 15137 \\
\hline
\end{tabular}

Como se observa, en las cuatro regiones de vieja colonización, las tasas de ocupación de un gran animal por hectárea dejan a los ovinos en el aire... y en Areco o en las estancias de cercanías, incluso sin tomar en cuenta a los ovinos, no alcanzamos a "meter" los vacunos y los yeguarizos a tasas de un animal por hectárea. Si hacemos el cálculo propuesto de otorgar 10 puntos a los vacunos/yeguarizos y 2 puntos a los ovinos -siempre por hectárea- Areco alcanza la cifra record de 32 puntos con 2 vacunos/yeguarizos y 6 ovinos por hectárea...

Lógicamente, la diferencia en las tasas de ocupación entre las áreas de más vieja colonización y las nuevas, no sólo está directamente ligada a las extensiones diferenciales de las propiedades, sino también está relacionada con los tipos de pastos: en las áreas más antiguas la mayor parte de los pastos son "blandos" o "tiernos" y en las nuevas el proceso de alteración de la pradera por efecto del continuo pastoreo está recién comenzando ${ }^{25}$. Pe-

nada que ver con la célebre enviada a Quiroga en 1834- Rosas describe los efectos de la sequía en estos términos: "Desde Morón asta este punto no hay mas qe. tierras, ganados vacunos y caballos muertos y por morir. Los ganados qe. no han podido por sus dueños ser sacados para los campos de la nueva frontera, los unos han perecido y los otros estan por morir...Felizmte. la mayor parte de los hacendados pudientes han podido sacar sus haciendas para los campos indicados. Las qe. han quedado al pareser son de pobres..." (Ratto, 1980: 113-114).

25 Hemos tratado este problema en Garavaglia, en prensa; uno de los primeros estudiosos que habló de este tema fue Charles Darwin cuando en 1832 realizó su viaje a caballo desde Carmen de Patagones y Bahía Blanca hacia Buenos Aires. Su sorpresa al descubrir el repentino mejoramiento de la pradera una vez que se atravesaba el Salado y sus atinados comentarios, en relación a la influencia de los grandes rumiantes en los cambios sucedidos 
ro, en las condiciones de producción de la época, es obvio que estas tasas están tocando ya lo que ha sido llamado "carrying capacity" ("capacidad de sustentación") por los ecólogos ${ }^{26}$ y basta que sobrevenga un período de fuerte sequía para que estas tasas caigan abruptamente. Los datos para los años 1830/1836 -para las áreas en que contamos con cifras- son bastante terminantes en este sentido.

\begin{tabular}{|llllll|}
\hline \multicolumn{5}{|c|}{ Cuadro 3 / Ocupación ganadera por regiones: } & $1830 / 1836$ \\
& Norte & Areco & Luján & Est.Cercs. & Sur I \\
superficie & 20592 & S/D & 13635 & 3172 & 34033 \\
número ests. & 6 & S/D & 8 & 2 & 10 \\
vac/yeguariz. & 13200 & S/D & 5192 & 642 & 12276 \\
ovinos & 3089 & S/D & 5471 & 2919 & 9318 \\
\hline
\end{tabular}

Mientras que el Sur I se mantiene estable (al igual que las estancias de cercanías, pero aquí la magra cantidad de establecimientos no permite sacar casi ninguna conclusión), las cifras para el Norte y sobre todo, para Luján muestran claramente los efectos de la sequía. Dado que la representatividad respectiva del Sur I y de Luján son altas, podemos verificar claramente los efectos diferenciales de esa sequía: mientras las tasas lujanenses descienden casi a la mitad, las del Sur, como decíamos, se mantienen en un mismo nivel. Estas cifras nos muestran claramente el accionar diferencial de la sequía en campos descargados y en los que ya han alcanzado o sobrepasado su capacidad de sustentación dadas las condiciones tecnológicas de la época ${ }^{27}$. Los datos para el período siguiente no hacen más que confirmar lo anterior.

en ella, constituyen el primer análisis científico de este fenómeno (ver Darwin, 1992); hay una excelente descripción realizada un poco más tarde entre nosotros, en Hernández، 1995: 29-31.

26 Es decir, la cantidad de individuos de una especie hervíbora que puede soportar un manto vegetal -en estado de palatabilidad y nutrición aceptable para esa especie-, durante un período determinado y sin afectar la producción forrajera de los períodos subsiguientes; ver Peek, 1980: 217-227.

27 Jorge Gelman ha estudiado unos censos ganaderos del período que muestran el estado de la ganadería en esos años, ver Gelman, 1996. 


\begin{tabular}{|lllllll|}
\hline \multicolumn{6}{c}{ Cuadro 4 / Ocupación ganadera por regiones: 1836/1852 } \\
& Norte & Areco & Luján & Est.Cercs. & Sur I & Sur II \\
superficie & 9068 & 5896 & 18808 & 3283 & 57813 & 185418 \\
número ests. & 6 & 6 & 8 & 4 & 15 & 12 \\
vaclyeguariz. & 16065 & 2971 & 21702 & 2974 & 33187 & 88468 \\
ovinos & 13190 & 1776 & 18556 & 4279 & 47308 & 16226 \\
\hline
\end{tabular}

Nuevamente (y la región Norte resulta impresionante en este sentido ${ }^{28}$ ) las tasas de ocupación pegan un salto y la ganadería se recupera en todas las regiones -con la notable excepción de Areco- mostrando cifras bastante altas. También observamos cómo en Sur / se superan las cifras de los dos períodos anteriores y ahora la nueva frontera ganadera se ha corrido a Sur II. Estos datos confirman muchas otras cosas que estamos ahora conociendo a partir de diversos trabajos en preparación ${ }^{29}$.

Debemos hacer ahora un ejercicio muy arriesgado pero necesario. Vamos a convertir los diversos valores utilizando la escala de promedios por hectárea que hemos propuesto antes (recordemos: 10 puntos para vacunos y yeguarizos y 2 puntos a los ovinos). Los datos para los distintos períodos están incluídos en el cuadro 5.

\begin{tabular}{|c|c|c|c|c|c|c|}
\hline \multicolumn{7}{|c|}{ Cuadro 5 / Ocupación ganadera } \\
\hline & Norte & Areco & Luján & Est.Cercs. & Sur I & Sur II \\
\hline \multirow[t]{2}{*}{$1816 / 29$} & 1,5 vac. & 2 vac. & $1,5 \mathrm{vac}$ & 2 vac. & 0,5 vac. & --.-.-- \\
\hline & 1 ovi. & 6 ovi. & 3 ovi. & 3,5 ovi. & 1 ovi. & -.----- \\
\hline valor & 17 & 32 & 21 & 27 & 7 & -n-m- \\
\hline \multirow[t]{2}{*}{$1830 / 36$} & $1 \mathrm{vac}$. & $S / D$ & $1 \mathrm{vac}$. & $0,5 \mathrm{vac}$ & 0,5 vac. & ------- \\
\hline & 2 ovi. & S/D & 1,5 ovi. & 1 ovi. & 1 ovi. & ---n- \\
\hline valor & 14 & & 13 & 7 & 7 & -n-m.- \\
\hline \multirow[t]{2}{*}{$1836 / 52$} & 2 vac. & 0,5 vac. & $1,5 \mathrm{vac}$ & $2 \mathrm{vac}$. & 1 vac. & $0,5 \mathrm{vac}$ \\
\hline & 6 ovi. & 1 ovi. & 5 ovi. & 3 ovi. & 2 ovi. & 1 ovi. \\
\hline valor & 32 & 7 & 25 & 26 & 12 & 7 \\
\hline
\end{tabular}

28 José Francisco Benítez, ganadero de San Nicolás, en los Antecedentes (1864:65) no olvida subrayar que “...en el Norte admiten los estancieros que caben cuatro mil cabezas de ganado vacuno en una legua cuadrada..."

29 Estamos realizando un estudio sobre los precios pecuarios hasta 1852, que muestra 14 
No tomemos a esta escala demasiado al pie de la letra, pues nuestro interés es únicamente encontrar una forma de hacer comparables los datos para los diversos períodos y las distintas regiones. Como decíamos antes, se comprueba claramente de qué modo la sequía de 1828/1832 va a significar un parteaguas en ese proceso de crecimiento en las tasas de ocupación, dado que éstas habían sobrepasado ya los límites de la capacidad de sustentación. Areco no parece haberse recuperado hasta fines del período que estamos analizando, en cambio el Norte y Luján retoman y sobrepasan las tasas de ocupación del primer período. A la vez, Sur / va lentamente creciendo -en especial, debido al incremento impresionante del stock ovino- en sus tasas de ocupación. Los mapas nos expresan gráficamente los datos de los diferentes valores para cada uno de los dos períodos extremos.

la caída de los precios en moneda constante en los años cuarenta, probablemente como efecto del crecimiento del stock ganadero; el trabajo sobre las exportaciones pecuarias que está terminando M. A. Rosal (Rosal, 1997) señala asimismo la importancia del incremento de las exportaciones en la década del cuarenta, reflejo indudable de ese crecimiento del stock de ganado. 
Tasas de ocupación: 1816-1829

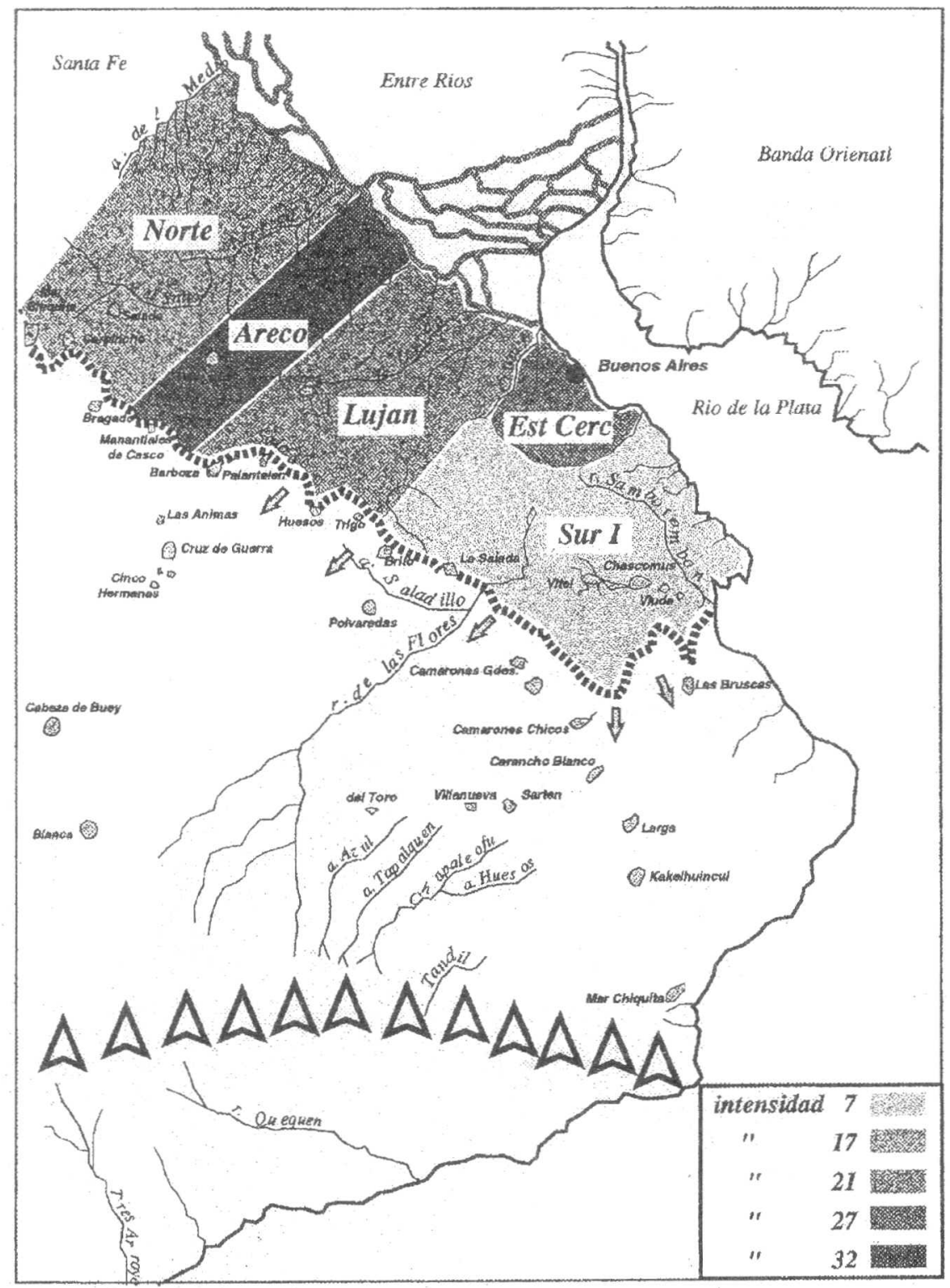

Según el mapa de Londres de 1824 
Tasas de ocupación: 1837-1852

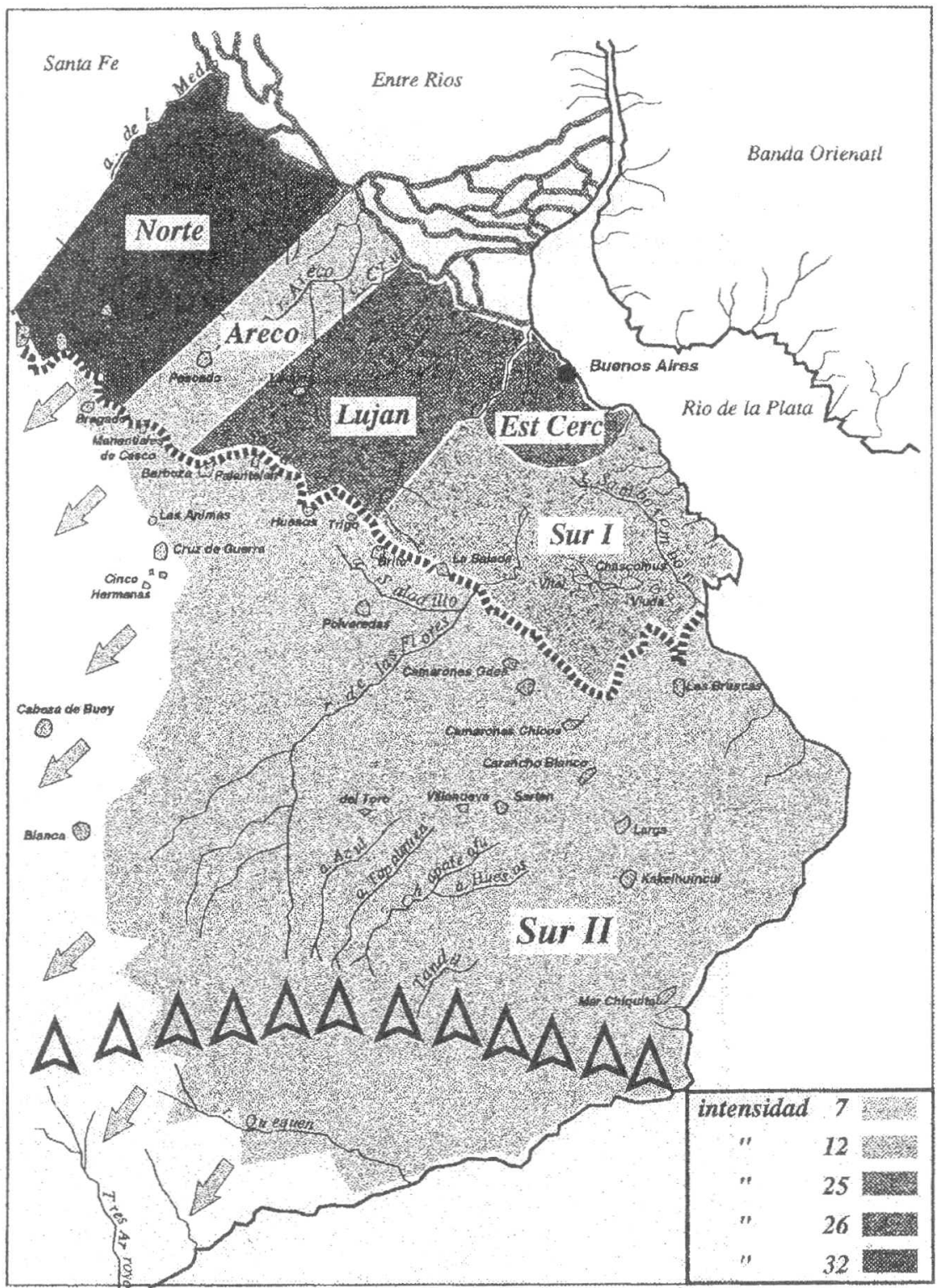

Según el mapa de Londres de 1824 
Unos pocos comentarios a estos mapas ${ }^{30}$ que casi hablan por sí mismos. Obviamente, en el período 1816/1829, la región Sur II no aparece con datos, pese a estar ya ocupada con rebaños desde hacía al menos quince años (en realidad, desde inicios del siglo XIX). Pero como no tenemos en la muestra seleccionada inventarios de propietarios que hayan muerto antes de 1829 -y ello es en cierto sentido lógico- se da ese vacío en nuestros datos. Como se advierte en ese primer período, Areco es el área de mayores tasas de ocupación relativamente hablando y justamente, será aquí en donde la caída sea más fuerte en el período 1837/1852. En este segundo período, el Norte es la zona de tasas más altas, seguido por Luján y las estancias de cercanías. Cuando Sur II aparece en forma abundante en los inventarios de la muestra, Sur I ha crecido ya en las tasas de ocupación, e incluso se podría hacer una división interna entre las más viejas áreas de Sur I (Ensenada, San Vicente y Magdalena) y las colonizadas hacia fines del XVIII, como Chascomús y Ranchos. En Sur II también se comienzan a diseñar diferencias con Dolores y Azul, relativamente más ocupadas por ganados y otros partidos, como Pila o Ajó, con tasas ganaderas realmente muy bajas.

\section{Conclusiones}

En marzo de 1856 se comienza a discutir en el Estado de Buenos Aires el proyecto de Código Rural. La discusión es muy importante porque refleja con claridad algunos de los conflictos más serios que oponían en ese entonces a los grandes hacendados y a los pequeños y medianos ganaderos (fueran éstos propietarios o no ${ }^{31}$ ). Uno de los aspectos esenciales de esta polémica tiene que ver con el papel que se asignaría a la pequeña propiedad pecuaria (la que correspondía a aquéllos que en su momento hemos llamado pastores) y justamente uno de los temas centrales de discusión gira alrededor de cuántos animales pueden ser mantenidos en una extensión determinada de terreno. Veámoslo con las propias palabras del texto:

30 Según el mapa de Londres de 1824, ver Carta de la provincia de Buenos Aires, publicada por D. Bartolomé Muñoz, en Londres, en diciembre de 1824, AGN, Colección PilladoBiedma (el lector notará que la representación de este mapa no es muy correcta, pero hemos preferido dejarlo tal cual para aprovechar todos sus topónimos de lagunas y otros pequeños cursos de agua).

31 Por supuesto, en su abrumadora mayoría las respuestas expresan la opinión de los grandes hacendados aun cuando parece haber algunos matices. 
"¿Convendria limitar en las estancias el número de cabezas de ganado en proporción del terreno, esto es fijar el maximun de ellas que pueda conservarse en una extensión dada de terreno? Parece que ha ofrecido serios inconvenientes la acumulación de animales en superficies cortas y desproporcionadas con su número, o sea en pequeñas estancias, produciendo asi el que los animales se derramen forzosamente en los campos inmediatos, o bien que las pequeñas estancias sean una ocasión para el hurto de los ganados de los vecinos. ${ }^{32}$ "

Las respuestas que los ganaderos consultados en cada partido darían a esta cuestión capital, son bastante variables y para no abusar de la paciencia del lector, tomaremos sólo las primeras, pero ellas son indicativas de la opinión generalizada entre los productores pecuarios:

"...en un terreno de cuatrocientas varas de frente y seis mil de fondo, pueden pastorearse doscientas cabezas de ganado vacuno, cien yeguarizo y seiscientos o novecientos lanares, sin embargo habrá que considerar la diferente calidad de los campos..." ${ }^{33}$

Esto quiere decir que en un terreno de 400 varas por 6000 (es decir de 180 ha. según nuestras equivalencias) habría 200 vacunos, 100 yeguarizos y de 600 a 900 lanares; ello nos daría una tasa de ocupación de 1.6 vacunos y yeguarizos y de 3.3 a 5 lanares por hectérea, lo que es casi exactamente la tasa real de ocupación de Luján en 1816/1829 y 1837/1852: 1.5. vacunos/yeguarizos y 3 lanares en el primer período y 1.5. vacunos/yeguarizos y 5 lanares en el segundo.

Manuel Villarino, otro de los productores solicitados, diría:

“...fijando un término medio; supongamos 1.500 vacunos, 2.000 lanar y 200 yeguariz en una suerte de estancia... " ${ }^{34}$

Si la suerte es de 3000 varas por 9000 y por lo tanto, de 2.024 ha., ello nos daría 0,8 vacunos/yeguarizos, más 1 lanar por hectárea y en este caso, esta tasa correspondería a un valor intermedio ideal para las regiones "nuevas" de Sur I y de Sur II en todos los perío-

32 Ver Antecedentes, 1864: 8.

33 Respuesta de Bernardo Gutierrez, Antecedentes, 1864: 25-26.

34 Ver respuesta de Manuel Villarino, Antecedentes, 1864: 37-38. 
dos aquí estudiados.

Otro de los ganaderos consultados no olvida mencionar que no se trata sólo de tomar en cuenta la superficie y la existencia o no de aguadas naturales, sino también el tipo de pastos y distingue claramente la cantidad de cabezas en función justamente del hecho de que los pastos fueran "tiernos" o "duros" 35 .

No podemos seguir con cada una de las respuestas ${ }^{36}$, pero es evidente que las cifras que aquí estamos presentando estaban en la cabeza de todos los que participaban en esta discusión y justamente, esas altas tasas de ocupación ganadera, eran uno de los temas centrales que preocupaban a los grandes hacendados en función de sus conflictos cotidianos con los pequeños y medianos propietarios. En las condiciones de producción de la época y con pocos campos alambrados, era obvio que los ganados de aquéllos que tuvieran campos muy sobrecargados, terminarían en algún momento pastoreando en las tierras de los propietarios adyacentes y esto, como resulta obvio a través de las discusiones acerca del futuro Código Rural, afectaba el derecho de propiedad ${ }^{37}$. Esta es una de las manifestaciones más claras de los conflictos de "clase" entre los grandes hacendados y los pastores. Conflicto que venía en realidad desde hacía mucho tiempo, pues puede ser rastreado al menos desde mediados del siglo XVIII, aunque con características diversas ${ }^{38}$.

Como se ve a través de nuestros datos, hay una tendencia bastante marcada entre el tamaño medio de las propiedades y las tasas de ocupación ganadera: a menor tamaño, tasas de ocupación más altas; ello indicaría que la dinámica de la pequeña y mediana propiedad pecuaria era bastante más eficaz de lo que muchas veces se supone ${ }^{39}$, aunque siempre estaba latente el riesgo de afectar durablemente -tal puede haber sido el caso de

35 Respuesta de Mariano Benítez, Antecedentes, 1864: 50.

36 En Antecedentes, 1864: 252, sección titulada "Indicaciones para el Código Rural" se termina por aconsejar una tasa de ocupación de 1.77 vacunos y yeguarizos y de 6 a casi 9 lanares por hectárea, lo que correspondería a las tasas más altas que hemos hallado nosotros gracias a los inventarios. Finalmente, el Código de 1864 en su artículo 6, deja totalmente librada al arbitrio de los propietarios esta materia.

37 Uno de los ganaderos que lo dice con mayor claridad es Matías Ramos Mexía, en Antecedentes, 1864: 54.

$38 \mathrm{Cfr}$. Garavaglia, en prensa. 
Areco- la capacidad de sustentación de sus campos en caso de una epizootia o, sobre todo, de una sequía de cierta importancia.

Creemos que las cifras presentadas aquí pueden ser de utilidad y en especial, si pensamos en la enorme masa de pastores no propietarios de la tierra (ya hemos visto que en algunas áreas, éstos correspondían a más de la mitad de los inventarios de la muestra), pues ellas nos permitirán ahora calcular tasas de ocupación ganadera más realistas que abarquen a los propietarios y a los no propietarios, tanto por períodos como por regiones, y podremos tener así una visión mucho más adecuada del estado de la economía agraria durante la época.

Paris, marzo de 1998.-

39 Uno de los ganaderos de San Nicolás que responde a las preguntas de la Comisión de Hacendados acerca del Código, no duda en afirmar que los pequeños rodeos permiten tasas de ocupación más altas (ver la respuesta de José Francisco Benítez, en Antecedentes, 1864: 65). 


\section{Bibliografia citada}

AMARAL, Samuel y J. M. GHIO (1990). "Diezmos y producción agraria. Buenos Aires, 17501800" En Revista de Historia Económica, VIII, 3, Madrid.

ANTECEDENTES (1864). Antecedentes y fundamentos del Proyecto de Código Rural (1856). Buenos Aires, Imprenta de Buenos Aires.

AZCUY AMEGHINO, E. y G. MARTINEZ DOUGNAC (1989). Tierra y ganado en la campaña de Buenos Aires según los Censos de Hacendados de 1789. Buenos Aires, Investigaciones, I.I.H.E.S.

BEAUMONT, J. A. B. (1957). Viajes por Buenos Aires, Entre Rios y la Banda Oriental (1826-1827). Buenos Aires, Hachette.

CANEDO, Mariana (1997). La tierra y la población en un área de temprana colonización. El Partido de los Arroyos, 1600-1850. Tesis de Doctorado, Universidad de Buenos Aires.

DARWIN, Charles (1992). Voyage d'un naturaliste autour du monde. Paris, La Découverte, vol. I (traducción del Diary of the Voyage of H.M.S. Beagle, New York, 1933).

FRADKIN, Raúl (1995). Según la costumbre del pays': costumbre y arriendo en Buenos Aires durante el siglo XVIII. Boletín del Instituto de Historia Argentina y Americana "Dr. E. Ravignani", 3ra. serie, 11, Buenos Aires.

GARAVAGLIA, J. C. (1993). "Las 'estancias' en la campaña de Buenos Aires. Los medios de producción" En Raúl Fradkin (editor) La historia agraria del Río de la Plata colonial: los establecimientos productivos. Buenos Aires, Centro Editor de América Latina.

GARAVAGLIA, J. C. (1997). Un siglo de 'estancias' en la campaña de Buenos Aires: 1751/1853, mimeo.

GARAVAGLIA, J. C. (en prensa). Pastores y labradores de Buenos Aires. Una historia agraria de la campaña bonaerense, 1700-1830. Buenos Aires, Ediciones de la Flor.

GELMAN, Jorge (1996). "Unos números sorprendentes. Cambio y continuidad en el mundo agrario bonaerense durante la primera mitad del siglo XIX" En Anuario IEHS, N¹1, Tandil.

GIBERTI, H. (1981). Historia económica de la ganaderia argentina. Buenos Aires, Ediciones Solar.

HERNANDEZ, José (1995). Instrucción del estanciero (1881). Buenos Aires, Editorial J. A. Roca. 
INFESTA, M. E. (1994). "Propiedad rural en la frontera. Azul, 1839". En Enrique M. Barba in memoriam. Estudios de Historia. La Plata, Edición Fundación Banco Municipal de La Plata.

INFORME (1958). "Informe del Señor Cónsul de Francia en Montevideo, M. Raymonde Baradere, al Ministerio de Relaciones Exteriores de Francia" (1834). En Revista Histórica, Museo Histórico Nacional, tomo XXVIII, Montevideo.

JURADO, José M. (1875). "La estancia en Buenos Aires". En Anales de la Sociedad Rural Argentina, tomo IX.

LATZINA, F. (1888). Geografía de la República Argentina. Buenos Aires, Lajouanne.

MUÑIZ, F. J. (s/f). Escritos cientificos. Buenos Aires, Jackson.

PEEK, J. (1980). "Natural Regulation of Ungulates (What Constitutes a Wilderness?)". En Wildlife Society Bulletin, Nº.

RATTO, M. E. (1980). "La posta de Figueroa". En Anales de la Junta de Estudios Históricos de San Antonio de Areco, I, San Antonio de Areco.

ROSAL, Miguel A. (1997). La exportación de cueros, lana y tasajo a través del Puerto de Buenos Aires, 1830-1854, mimeo. 